\title{
Manifestation of the Jupiter's synodic period in the solar wind, interplanetary magnetic field and geophysical parameters
}

\author{
S. N. Samsonov ${ }^{1}$ and N. G. Skryabin ${ }^{1}$ \\ ${ }^{1}$ Yu. G. Shafer Institute of Cosmophysical Research and Aeronomy, SB RAS, \\ 677980, Yakutsk, Russia \\ email: s_samsonov@ikfia.ysn.ru
}

\begin{abstract}
Studying by the authors of paper of solar wind parameters, namely: density, speed and temperature and also a module of interplanetary magnetic field (IMF) intensity has allowed to find out in them fluctuations with the period of 399 days. From references it is known that this period coincidence with the synodic period of Jupiter. So long as close by the given period another source of such fluctuations is not known we have assumed that fluctuations with the period of 399 days are fluctuations with the synodic period of Jupiter. The change of the solar wind plasma parameters and IMF intensity can lead to the change of the Earth's magnetic field parameters and, as a consequence, to the change of charged particle fluxes in the Earth's magnetosphere. On this assumption the IMF intensity in the Earth's vicinity, geomagnetic disturbance (Kp-index) and riometer absorption for the years of 1986-1996 have been analyzed. The analysis of the data has shown the presence of certain changes of these physical parameters with the period of 399 days. When the Earth and Jupiter were found to be on the same magnetic field line, the IMF intensity was decreasing up to $3.0 \pm 0.57 \%$, the geomagnetic activity and riometer absorption were decreasing up to $5.2 \pm 1.46 \%$ and $9.4 \pm 2.63 \%$, respectively.
\end{abstract}

Keywords. Sun: activity, solar wind, solar system: general

\section{Introduction}

It has been established in the early (for examples, Hill, Carbary \& Dessler (1974) and modern (for examples, Ferreira et al. 2001) researches that Jupiter is a powerful source of charged particles. Hill, Carbary \& Dessler (1974) using the observations of energetic electrons in the heliosphere from 1965 to 1974 showed that the increase of electron intensity is observed when the Earth appears to be close or crosses the IMF field line connecting the Sun and the Jupiter. But in works by Skryabin et al. (2005) and Timofeev et al. (2007) it was found that the variations with the Jupiter's synodic period of 399 days are observed in the plasma density of the solar wind, the intensity of cosmic rays and energetic electrons on the Earth's orbit.

The most favorable conditions for the propagation of the electrons from Jupiter towards the Earth are realized during their movement along the IMF field line. McDonald \& Trainor (1976) showed that such conditions are practically realized every 13 months and last for 1-3 months.

So the authors of paper have decided to check up the following fact: whether there are such fluctuations in the solar wind parameters and interplanetary magnetic field.

The purpose of this work is to search for a variation with a synodic period in the IMF, geomagnetic disturbance index and riometer absorption. 


\section{Experimental data and results}

Riometer measurements were carried on in Tixie from 1986 to 1996 . The daily values of the absorption occurrences were used as riometer data. The event of absorption was considered to have taken place at the given moment if its intensity was $>0.3 \mathrm{~dB}$ and the absorption lasted not less than 10 minutes. The cases caused by proton events and solar X-ray bursts were excluded from the experimental data.

The daily data of absorption occurrence frequency prepared in such a way are shown on the top panel (A) of Fig.1a. The ordinates axis shows the quantity of hours per day when the absorption was observed. The abscissa axis shows the ordinal number of the day beginning from January 1, 1986. The rare gaps in the observations "were restored" on the basis of observations made at neighbouring stations (Sokolov et al. 1988).

To exclude the short-period changes from the experimental data the method of smoothing was applied as a method of moving average with the period of averaging of 200 days. The smoothed experimental data of riometer absorption are given on the second panel (N) of Fig.1a. The verticals on this panel correspond to the moments of the opposition of the Earth and Jupiter. The axis of ordinates shows the daily frequency of occurrence of absorption, the abscissa axis - a through numbering of the days from January 1, 1986 to December 31, 1996 (4018 days). On the panels of Kp and IMF (OMNIWeb data) the data of daily $\mathrm{Kp}$-index of the geomagnetic disturbance and IMF intensity module after smoothing are presented.

Then the experimental data given in Fig.1a were divided into 10 intervals equal to the average synodic period of Jupiter and after that they were processed using a superposed epoch technique. The results of such processing are presented in Fig.1b (for better clearness two periods are shown). In this Figure the solid verticals correspond to the moment of the opposition of the Earth and Jupiter, the dotted lines - to the expected location of Jupiter, the Earth and the Sun on the same IMF line. This moment comes, at an average, 156 days before the next opposition or 243 days after it. According to Skryabin et al. (2001) the average synodic period of Jupiter in the 21-st solar activity cycle is equal to $401 \pm 7$ days changing from 372 to 437 days.

From Fig.1b it follows that the effect of Jupiter in the riometer absorption is 0,8 events a day or $9,4 \%$ from the average value equal to 8.5 events per day for the whole period of observation; in the geomagnetic disturbance (K-index) the effect is 1.0 or $5,2 \%$ from the average value equal to 19.3 ; in the IMF the effect is equal to $0.2 \mathrm{nT}$ or $3,0 \%$ from the average value equal to $6.74 \mathrm{nT}$.

Fig.1c shows distributions of casual deviations from the results of application of superposed epoch technique. By these distributions the errors shown in Fig.1b have been determined using standard procedures. The amplitude distributions appear to be really similar to the law of normal distribution. That's why we consider that the errors in Fig.1b are caused by casual deviations and are equal to: $\sigma=0.165$ for the riometer absorption data and $\sigma=0.20$ for the Kp-index and $\sigma=0.027$ for the IMF.

\section{Conclusion}

The results presented above have allowed to find out the fluctuations with the synodic period of Jupiter in the IMF intensity, geomagnetic ctivity and riometer absorption in addition to the fluctuations with the synodic period of Jupiter in the solar wind parameters. The value of Jupiter's effect for the considered period accounts for $3.0 \pm 0.57 \%$ in the IMF, $5.2 \pm 1.46 \%$ in the geomagnetic activity and $9.4 \pm 2.63 \%$ in the riometer absorption. 

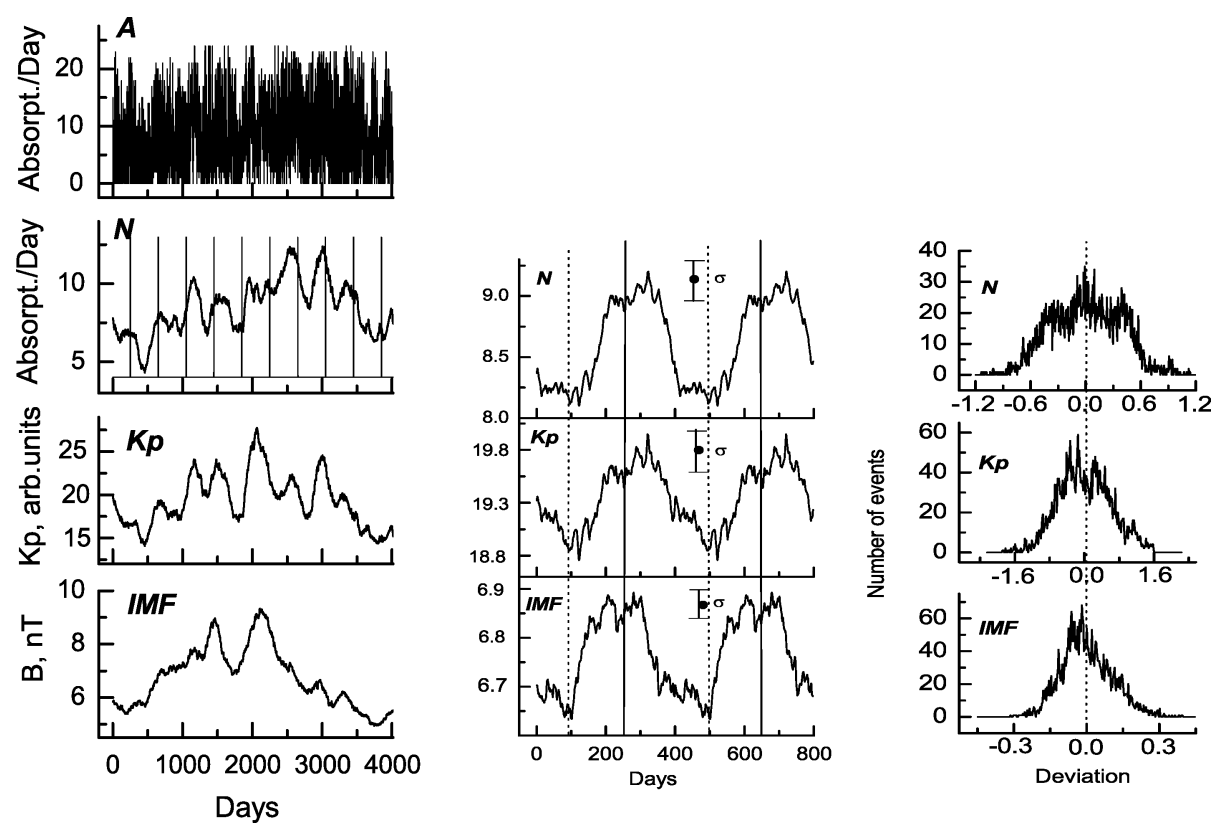

Figure 1. a) Raw riometer data (A) and smoothed data of the riometer absorption (N), Kp-index (Kp) and interplanetary magnetic field module (IMF); b) Variations of the riometer absorption (N), Kp-index (Kp) and IMF module (IMF) with the synodic period of Jupiter. $\sigma$ - is the root-mean-square deviation.; c) Amplitude distribution of the following deviations for the riometer absorption $(\mathrm{N})$, the Kp-index $(\mathrm{Kp})$ and the IMF module (IMF).

The work was partly financed by RFBR (grants N 09-02-98505-p_vostok_a and 09-0200284-a and the RAS Presidium Program for the basic research N16, part 3.

\section{References}

Ferreira, S. E. S., Potgieter, M. S., Burger, R. A., Heber, B., Fichtner, H., \& Lopate, C. 2001, Geophys. Res., 106, 29313

Hill, T. W., Carbary, J. F., \& Dessler, A. J. 1974, Geophys. Res. Lett., 1, 333

McDonald, F. B. \& Trainor, J. H. 1976, in: T. Gerels (eds.), Jupiter: Studies of the interior, atmosphere, magnetosphere and satellites Tucson: University of Arizona Press, p. 961

Skryabin, N. G., Bezrodnykh, I. P., Plotnikov, I. Ya., \& Ivanov, O. P. 2001, Geomagnetism and Aeronomy, 41, 432

Skryabin, N. G., Timofeev, V. E., Miroshnichenko, L. I., \& Samsonov, S.N. 2005, Astronomy Letters, 31, 832

Sokolov, V. D., Samsonov, S. N., Ivanov, O. P., Yarygin, L. S., Vasil'ev, N. I., \& Filippovich, V. N. 1988, Observations of artificial celestial bodies (in Russian), 84, 206

OMNIWeb data, http://omniweb.gsfc.nasa.gov

Timofeev, V. E., Miroshnichenko, L. I., Samsonov, S. N., \& Skryabin, N. G. 2007, Astronomy Letters, 33, 63 